\title{
Correction to: The genomics of invasion: characterization of red lionfish (Pterois volitans) populations from the native and introduced ranges
}

\author{
M. O. Burford Reiskind (1) - E. M. X. Reed - A. Elias • J. J. Giacomini • \\ A. F. McNear - J. Nieuwsma - G. A. Parker - R. B. Roberts - R. E. Rossi • \\ C. N. Stephenson · J. L. Stevens • B. E. Williams
}

Published online: 8 May 2019

(C) Springer Nature Switzerland AG 2019

\section{Correction to: Biol Invasions \\ https://doi.org/10.1007/s10530-019-01992-0}

In the original publication of this article, Figs. 2 and 3 were published incorrectly.

Figure 2 should be the STRUCTURE plot that includes 8 populations (see figure). It should not be a second copy of the map that is found in Fig. 1.
Figure 3 should be two parts: 3a, which is two STRUCTURE plots for the two population settings, and $3 b$, which is a DAPC plot (see figure). They should not be a single STRUCTURE plot as in the online version.

The corrected Figs. 2 and 3 are given in this correction.

The original article can be found online at https:// doi.org/10.1007/s10530-019-01992-0.

M. O. Burford Reiskind $(\bowtie) \cdot$ E. M. X. Reed .

J. J. Giacomini - A. F. McNear · R. E. Rossi .

J. L. Stevens

Department of Applied Ecology, North Carolina State

University, Campus Box 7617, Raleigh, NC 27695, USA

e-mail: mbreiski@ncsu.edu

A. Elias · J. Nieuwsma - G. A. Parker .

R. B. Roberts - C. N. Stephenson - B. E. Williams

Department of Biological Sciences, North Carolina State

University, Raleigh, NC 27695, USA

G. A. Parker - R. B. Roberts

WM Keck Center for Behavioral Biology, North Carolina

State University, Raleigh, NC 27695, USA 


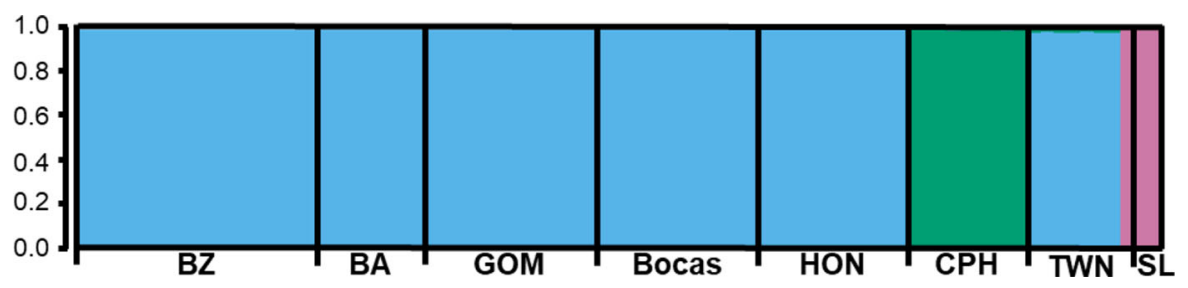

Fig. 2 Estimated population structure from STRUCTURE analysis for eight lionfish populations (excluding $P$. antennata) with a $K=3$ using 52,742 polymorphic loci. Each color corresponds to a genetic cluster and each individual is represented by a vertical bar. Structure harvester supports a $K=3$ Here $\mathrm{BZ}=$ Belize, $\mathrm{BA}=$ Bahamas, $\mathrm{GOM}=$ Gulf of Mexico, Bocas $=$ Panama, HON $=$ Honduras, $\mathrm{CPH}=$ Philippines, $\mathrm{TWN}=$ Taiwan, and $\mathrm{SL}=$ Sri Lanka. BZ, BA, GOM,
Bocas, and $\mathrm{HON}$ are from the Lionfish invasive range, $\mathrm{CPH}$ and TWN are Lionfish from the native range identified in the field as $P$. volitans, and SR are Lionfish from the native range identified as $P$. miles. All invasive populations and TWN (except for one individual) were assigned to one cluster, SL and one TWN individual were assigned to a second, and $\mathrm{CPH}$ individuals comprised the third
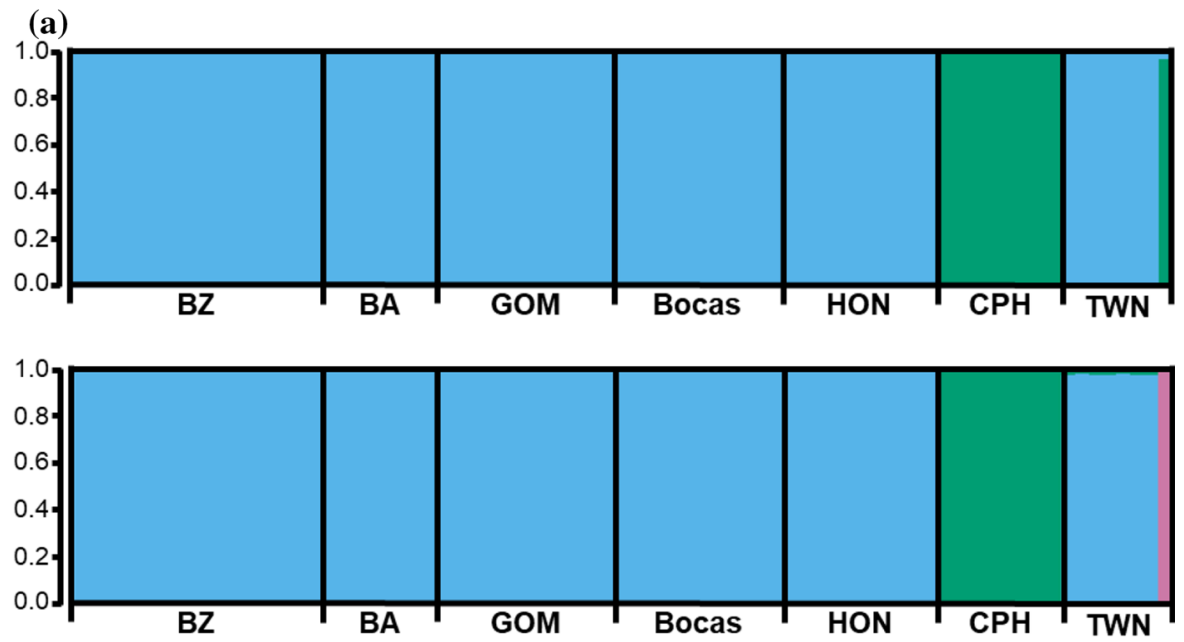

(b)

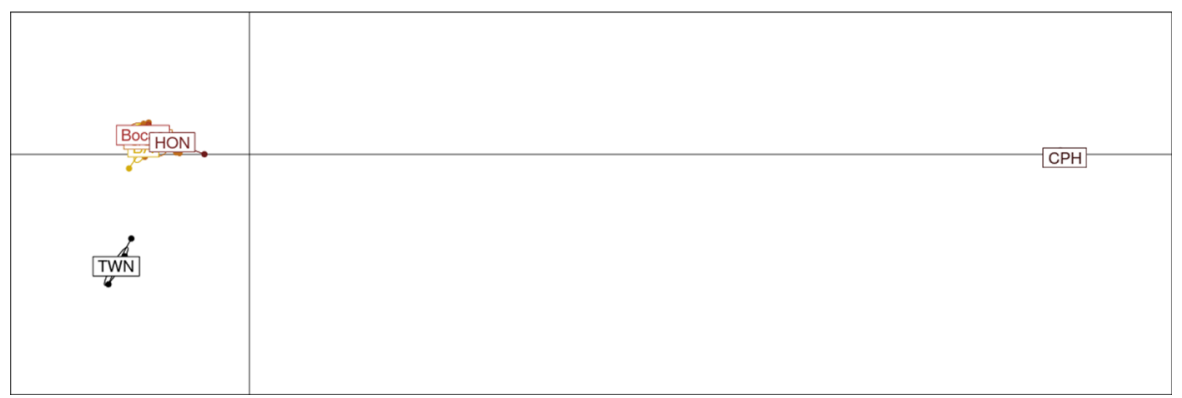

Fig. 3 Graphical representation of the seven putative $P$. volitans populations using 44,190 polymorphic loci, where the first five populations are from the invasive range $(\mathrm{BZ}=$ Belize, $\mathrm{BA}=$ Bahamas, $\mathrm{GOM}=$ Gulf of Mexico, Bocas = Panama, $\mathrm{HON}=$ Honduras) and the last two are from the native range $(\mathrm{CPH}=$ Philippines, $\mathrm{TWN}=$ Taiwan $)$. a Visual output from STRUCTURE analysis with a $K=2$ and $K=3$. Structure harvester supported a $K=2$, and we included the representation for three clusters to highlight the putatively misidentified individual from Taiwan (TWN). All invasive populations and
TWN except for the one individual were assigned to the same cluster. CPH individuals were all assigned to a second cluster, to which the TWN individual was also assigned when $K=2$. When $K=3$, the one TWN individual was unique from all others. b Visual output using Discriminant Analysis of Principal Components (DAPC) analysis with 10 principal components, chosen to avoid overfitting the data, and cross-validation of 95 iterations. The DAPC analysis shows that CPH is genetically distinct from the invasive and TWN populations. The invaded range populations overlap each other 
Publisher's Note Springer Nature remains neutral with regard to jurisdictional claims in published maps and institutional affiliations. 